\title{
Caracterização da Uveíte na Espondilite Anquilosante $e^{(*)}$
}

\section{Characterization of Uveitis in Ankylosing Spondylitis}

\author{
Percival Degrava Sampaio-Barros ${ }^{(1)}$, Manoel Barros Bértolo ${ }^{(2)}$ e Adil Muhib Samara ${ }^{(3)}$
}

\section{RESUMO}

Objetivo: analisar a apresentação clínica e a evolução da uveíte em uma população de 207 pacientes com espondilite anquilosante (EA). Métodos: estudo retrospectivo (1988-2001) analisando 207 pacientes com o diagnóstico de EA segundo os critérios de Nova York modificados. Todos apresentavam investigação clínica (envolvimento axial e periférico, entesopatias, manifestações extraarticulares) e radiológica (sacroilíaca, coluna lombar, dorsal e cervical) completas, além da pesquisa do HLA-B27 (com seus respectivos alelos, quando possível). Os dados foram comparados com a presença de uveíte durante o período de seguimento dos pacientes. Resultados: trinta pacientes (14,5\%) apresentaram 55 episódios de uveíte anterior aguda unilateral durante o seguimento ambulatorial. Vinte e sete pacientes encontravam-se em atividade articular da EA no momento da crise de uveíte, enquanto três pacientes encontravam-se inativos do ponto de vista articular. Um único paciente, em tratamento de tuberculose pulmonar, apresentou um episódio de uveíte posterior. Entre os pacientes com uveíte, houve predomínio de sexo masculino (82,6\%), raça caucasóide (77,8\%), início da EA na idade adulta (83,1\%), HLA-B27 positivo $(79,2 \%)$, sem história familiar de EA $(84,5 \%)$. Houve associação estatística entre uveíte e idade de início juvenil $(\mathrm{p}=0,0094)$ e entesopatias aquileana $(\mathrm{p}=0,0003)$ e plantar $(\mathrm{p}=0,0067)$. Nenhum paciente apresentou seqüela ocular grave da uveíte. Dezesseis pacientes fizeram uso de sulfassalazina $(1,0 \mathrm{~g} / \mathrm{dia}$ a 2,0 g/dia), por prazo mínimo de seis meses. Conclusões: a uveíte anterior aguda foi comum na evolução da EA nesta casuística, estando associada preferencialmente à EA de início juvenil e ao acometimento articular entesopático de membros inferiores.

Palavras-chave: espondilite anquilosante, uveíte, HLA-B27, entesopatias, espondilite anquilosante juvenil.

\section{ABSTRACT}

Objective: To analyze the clinical picture and outcome of uveitis in a series of 207 patients with ankylosing spondylitis (AS). Methods: Retrospective study (1988-2001) analyzing 207 patients with diagnosis of AS according to the modified New York criteria. All patients were submited to complete clinical (axial and peripheral involvement, heel enthesopathies, extraarticular manifestations) and radiological (sacroiliac, lumbar, dorsal and cervical spine) investigation, HLA-B27 evaluation (and respective alleles, whenever possible), were searched. These data were compared with the occurrence of uveitis during the follow-up of the AS patients. Results: Thirty patients (14.5\%) presented 55 episodes of acute unilateral anterior uveitis. Twenty seven patients had active articular disease at the moment of the uveitis crisis, while three patients were inactive. One patient, in treatment of lung tuberculosis, presented a single episode of posterior uveitis. Among the uveitis in AS patients, there was predominance of male sex (82.6\%), Caucasoid race (77.8\%), adult-onset AS (83.1\%), positive HLA-B27 (79.2\%) and absence of familial history of AS (84.5\%). There was statistical association between uveitis and juvenile-onset AS ( $p=0.0094)$ and achillean $(p=0.0003)$ and plantar $(p=0.0067)$ enthesopathies. No patient presented severe ophthalmologic sequelae of uveitis. Sulfasalazine (1 to $2 \mathrm{~g}$, daily) was prescribed to 16 patients, for a minimum period of six months. Conclusions: Acute anterior uveitis was common in the follow-up of AS patients in this casuistic, associated to the juvenile-onset of the disease and to the enthesophatic involvement of the lower limbs.

Keywords: ankylosing spondylitis, uveitis, HLA-B27, enthesopathies.

\footnotetext{
* Trabalho realizado pela Disciplina de Reumatologia do Departamento de Clínica Médica da Faculdade de Ciências Médicas da Universidade Estadual de Campinas (FCM-UNICAMP), Campinas, SP. Este trabalho contou com recursos dos Fundos Remanescentes da Sociedade Brasileira de Reumatologia Recebido em 20/02/2003. Aprovado, após revisão, em 12/06/2003.

1. Reumatologista-Assistente. Doutor em Medicina. FCM-UNICAMP.

2. Professor Assistente-Doutor. FCM-UNICAMP.

3. Professor Titular. FCM-UNICAMP.
}

Endereço para correspondência: Percival D. Sampaio-Barros. Rua Dr. Quirino, 1001, ap. 24, Centro, CEP 13015-081, Campinas, SP. E-mail: psbarros@fcm.unicamp.br 


\section{INTRODUÇÃO}

Manifestações oftalmológicas são comuns em diversas doenças reumáticas ${ }^{(1)}$. Entre estas manifestações, as uveítes costumam ser importantes porque muitas vezes representam a manifestação inicial de diversas patologias sistêmicas ${ }^{(2-7)}$. A uveíte anterior é referida com freqüência nas espondiloartropatias soronegativas ${ }^{(8)}$ e é considerada a manifestação extra-articular mais freqüente da espondilite anquilosante $(\mathrm{EA})^{(9)}$. Costuma ser aguda, unilateral, recorrente (com crises acometendo geralmente o olho contralateral) e autolimitada (com duração de até dois a três meses), podendo afetar até $40 \%$ dos pacientes espondilíticos em um seguimento de longo prazo $^{(8-10)}$. Também é freqüente a associação com a presença do HLA-B27 $7^{(11)}$, que é considerado um fator prognóstico da associação da uveíte anterior com manifestações osteoarticulares ${ }^{(12)}$. $\mathrm{O}$ único estudo avaliando os alelos do HLA-B27 em pacientes com uveíte anterior mostrou uma maior freqüência do HLA-B*270 $5^{(13)}$. Outros antígenos de histocompatibilidade associados com a uveíte anterior são o HLA-DR ${ }^{(14)}$, DR1 e DR12(13).

No Brasil, Carvalho et al. ${ }^{(15)}$, estudando 100 pacientes com uveíte não-granulomatosa, observaram que 38 pacientes apresentavam uma doença do grupo das espondiloartropatias soronegativas como doença de base e que os indivíduos HLA-B27 positivos com uveíte apresentavam chance 3,8 vezes maior de cursar com uma espondiloartropatia soronegativa. Já Trevisani et al. ${ }^{(16)}$, avaliando 34 pacientes HLA-B27 positivos e 23 HLA-B27 negativos, observaram que anticorpos específicos contra antígenos da íris foram igualmente detectados em ambos os grupos. No presente trabalho, os autores analisaram a apresentação clínica e a evolução dos casos de uveíte em uma população de 207 pacientes com EA.

\section{PACIENTES E MÉTODOS}

Os autores realizaram um estudo retrospectivo analisando a frequiência e a evolução clínica das crises de uveíte em uma casuística de 207 pacientes com diagnóstico de EA segundo os critérios de Nova York modificados ${ }^{(17)}$, acompanhados no ambulatório de reumatologia do Hospital das Clínicas da Unicamp, no período entre 1988 e 2001. Todos apresentavam investigação clínica e radiológica completas.

A avaliação clínica incluiu a pesquisa dos sintomas iniciais e da presença de envolvimento axial e periférico, entesopatias (aquileana e/ou plantar) e manifestações extra-articulares. A investigação radiológica incluiu a avaliação de articulações sacroilíacas, coluna lombar, dorsal e cervical. O HLA-B27 foi pesquisado em todos os pacientes, sendo também pesquisados os respectivos alelos (HLA-B*2701 a $B^{*} 2720$ ), por meio da reação de cadeia de polimerase utilizando-se primers específicos de alta resolução (Dynal, Oslo, Noruega), nos casos em que havia DNA estocado. Os dados foram comparados com a presença de uveíte durante o período de seguimento dos pacientes; também foi analisada a terapêutica utilizada no aparecimento da crise de uveíte.

\section{ANÁLISE ESTATÍSTICA}

Os testes do qui-quadrado e exato de Fisher foram utilizados para verificar a associação das variáveis de interesse; valores de $\mathrm{p} \leq 0,05$ foram considerados estatisticamente significativos.

\section{RESULTADOS}

Foram detectados 56 episódios de uveíte durante o período de estudo. Trinta pacientes $(14,5 \%)$ apresentaram 55 episódios de uveíte anterior aguda unilateral no período de seguimento do estudo. Um paciente espondilítico, com tuberculose pulmonar em início de tratamento, apresentou um único episódio de uveíte posterior; interpretada como manifestação da tuberculose, e apresentou remissão após tratamento com esquema tríplice. Entre as características epidemiológicas dos pacientes com uveíte, houve predomínio de sexo masculino (82,6\%), raça caucasóide (77,8\%), início da EA na idade adulta $(83,1 \%)$, HLA-B27 positivo $(79,2 \%)$, sem história familiar de EA $(84,5 \%)$. Verificou-se associação estatística entre uveíte anterior e idade de início juvenil $(p=0,0094)$. Embora 27 dos 30 pacientes que apresentaram uveíte anterior fossem HLA-B27 positivo, os valores não foram estatisticamente significativos $(\mathrm{p}=0,1157) ;$ os três pacientes HLA-B27 negativo não apresentaram recorrência do episódio de uveíte. Entre os 164 pacientes HLA-B27 positivo, 98 pacientes tiveram testado os respectivos alelos, sendo $90 \mathrm{com} \mathrm{B}^{*} 2705$, sete com $B^{*} 2702$ e um com $B^{*} 2713$; todos os 16 pacientes espondilíticos HLA-B27 positivos que apresentaram uveíte anterior e que foram testados para os alelos do B27 apresentaram $B^{*} 2705$. A média de idade de início da uveíte foi semelhante nos dois grupos $(22,87 \pm 9,77$ anos no grupo com uveíte contra 25,45 \pm 9,21 anos no grupo sem uveíte), enquanto a média de tempo de doença foi maior no grupo com uveíte $(20,23 \pm 9,05$ anos, contra 15,08 \pm 9,59 anos no grupo sem uveíte; $\mathrm{p}=0,0008)$. 
Quanto às manifestações articulares axiais, não foi observado associação estatística entre os dois grupos. Com relação às manifestações articulares extra-axiais, notou-se correlação estatística entre uveíte anterior e entesopatias aquileana $(\mathrm{p}=0,0003)$ e plantar $(\mathrm{p}=0,0067)$.

Vinte e sete pacientes encontravam-se em atividade articular da EA no momento da crise de uveíte, enquanto três pacientes encontravam-se inativos do ponto de vista articular. As crises tiveram duração média de três semanas. Nenhum paciente apresentou seqüela ocular grave das crises de uveíte; quatro pacientes tiveram de ser submetidos à cirurgia oftalmológica, sendo dois por glaucoma e dois para a retirada de sinéquias, todos com boa evolução. Optouse pelo uso de sulfassalazina $(1,0 \mathrm{~g} /$ dia a $2,0 \mathrm{~g} / \mathrm{dia})$, por prazo mínimo de seis meses, independentemente da atividade articular, em 16 pacientes (todos eram HLA-B27 positivos, incluindo 9 dos 11 pacientes com mais de uma crise durante o período de seguimento). Seis pacientes que apresentaram mais de duas crises de uveíte anterior e fizeram uso de sulfassalazina por período mínimo de um ano não apresentaram recorrência da uveíte durante o período de uso da droga.

\section{DISCUSSÃO}

A uveíte anterior é a manifestação extra-articular mais freqüentemente observada na $\mathrm{EA}^{(8-10)}$. Em casuística recentemente publicada, avaliando padrões de doença num grupo de 147 pacientes com EA acompanhados na Disciplina de Reumatologia da Unicamp, a uveíte anterior foi referida por $14,3 \%$ dos pacientes ${ }^{(18)}$. Este estudo aumentou o número de pacientes avaliados, analisou a presença dos alelos do HLA-B27 e a evolução clínica desses casos de uveíte.

Uveíte anterior aguda unilateral foi referida por 14,5\% dos pacientes estudados que apresentaram 55 episódios durante o período de seguimento. Não houve diferença estatística quanto a sexo, raça e média de idade de início entre os pacientes com e sem uveíte. No entanto, a uveíte anterior apresentou associação estatística com o início juvenil da EA (abaixo de 16 anos), que caracteristicamente apresenta maior prevalência de manifestações extra-axiais no seguimento a longo prazo $^{(9)}$. Já a uveíte posterior confirmou ser manifestação extremamente rara na EA, estando presente em um único caso, associado à tuberculose pulmonar.
O HLA-B27 é considerado um fator prognóstico de surgimento de uveíte no seguimento de pacientes com $\mathrm{EA}^{(19)}$. No presente estudo, 27 dos 30 pacientes que apresentaram uveíte anterior eram HLA-B27 positivo, mas o resultado não foi considerado estatisticamente significativo; quanto aos três pacientes HLA-B27 negativo com uveíte, foi possível observar que manifestaram uma única crise autolimitada e não necessitaram de terapêutica posterior com sulfassalazina. Com relação aos alelos do HLA-B27, foi observado somente o alelo B*2705 nos pacientes com uveíte anterior, semelhante ao que é observado nas populações predominantemente caucasóides ${ }^{(20,21)}$ e na população brasileira miscigenada ${ }^{(22)}$.

O tratamento das uveítes pela perspectiva de reumatologistas foi extensamente revisado recentemente ${ }^{(23)}$. O uso da sulfassalazina é considerado poupador da ocorrência de crises de uveíte anterior em pacientes com EA ${ }^{(24,25)}$. Na experiência dos autores, a sulfassalazina constitui-se uma boa indicação nos casos de uveíte anterior aguda recorrente na EA, já que a grande maioria das crises ocorre durante o período de atividade da doença e a sulfassalazina é uma boa droga no tratamento da EA, principalmente nos pacientes com componente periférico associado ${ }^{(26,27)}$. No presente trabalho, pode-se observar uma diminuição do número de crises após a introdução da sulfassalazina em vários pacientes, embora a natureza retrospectiva do trabalho não permitisse um estudo estatístico mais elaborado do assunto. $\mathrm{O}$ uso do metotrexato, outra droga de base comumente utilizada na EA não está associado à diminuição do número de crises de uveíte $^{(23,28)}$. Atualmente, a grande perspectiva no tratamento da EA é o uso de drogas inibidoras do fator de necrose tumoral, como o infliximab ${ }^{(29,30)}$ e o etanercept ${ }^{(31)}$; o uso destas drogas no tratamento de casos de uveíte refratária tem mostrado resultados iniciais promissores ${ }^{(32,33)}$.

Concluindo, a uveíte anterior foi comum na evolução da EA nesta casuística, estando associada preferencialmente à EA de início juvenil e ao acometimento articular entesopático de membros inferiores.

\section{AGRADECIMENTOS}

Os autores gentilmente agradecem a Andréia Semolini e Helymar Costa Machado pela análise estatística deste trabalho. 


\section{REFERÊNCIAS}

1. Hamideh F, Prete PE. Ophthalmologic manifestations of rheumatic diseases. Semin Arthritis Rheum 2001;30:217-41.

2. Rosenbaum JT. An algorithm for the systemic evaluation of patients with uveitis: Guidelines for the consultant. Semin Arthritis Rheum 1990;19:248-57.

3. Rothova A, Buitenhuis HJ, Meenken C, et al. Uveitis and systemic disease. Br J Ophthalmol 1992;76:137-41.

4. McCannel CA, Holland GN, Helm CJ, et al. Causes of uveitis in the general practice of ophthalmology. Am J Ophthalmol 1996;121:35-46.

5. Tay-Kearney ML, Schwam BL, Lowder C, et al. Clinical features and associated systemic diseases of HLA-B27 uveitis. Am J Ophthalmol 1996;121:47-56.

6. Bañares A, Jover JA, Fernández-Gutiérrez B, et al. Patterns of uveitis as a guide in making rheumatologic and immunologic diagnoses. Arthritis Rheum 1997;40:358-70.

7. Kotaniemi K, Aho K, Kotaniemi A. Uveitis as a cause of visual loss in arthritides and comparable conditions. J Rheumatol 2001; 28:309-12.

8. Bañares A, Hernández-Garcia C, Fernández-Gutiérrez B, Jover JA. Eye involvement in the spondyloarthropathies. Rheum Dis Clin North Am 1998;24:771-84.

9. Khan MA. Ankylosing spondylitis: clinical aspects. Em Calin A, Taurog JD (Editores): The Spondylarthritides. Oxford, Oxford University Press, 1998; pp. 27-40.

10. Rosenbaum JT. Acute anterior uveitis and spondyloarthropathies. Rheum Dis Clin North Am 1992;18:143-51.

11. Martin TM, Smith JR, Rosenbaum JT. Anterior uveitis: current concepts of pathogenesis and interactions with the spondyloarthropathies. Curr Opin Rheumatol 2002;14:337-41.

12. Linssen A, Meenken C. Outcomes of HLA-B27-positive and HLAB27-negative acute anterior uveitis. Am J Ophthalmol 1995; 120:351-61.

13. Konno Y, Numaga J, Tsuchiya N, et al. HLA-B27 subtypes and HLA class II alleles in Japanese patients with anterior uveitis. Invest Ophthalmol Vis Sci 1999;40:1838-44.

14. Islam SMM, Numaga J, Fujino Y, et al. HLA-DR 8 and acute anterior uveitis in ankylosing spondylitis. Arthritis Rheum 1995;38:547-50.

15. Carvalho MAP, Campos WR, Araújo CAA, Lacerda RR, Oréfice F. Uveítes anteriores não granulomatosas, espondiloartropatias e HLA-B27. Rev Bras Reumatol 1999;39:195-202.

16. Trevisani VF, Mattos KT, Esteves RF, Olialves SM, Andrade LE. Autoantibodies specificity in acute anterior uveitis according to the presence of the HLA-B27 allele. Ocul Immunol Inflamm 2001;9:231-42.

17. Van der Linden S, Valkenburg HA, Cats A. Evaluation of diagnostic criteria for ankylosing spondylitis: a proposal for modification of the New York criteria. Arthritis Rheum 1984;27:361-8.
18. Sampaio-Barros PD, Bértolo MB, Kraemer MHS, Marques Neto JF, Samara AM. Primary ankylosing spondylitis: Patterns of disease in a group of 147 Brazilian patients. J Rheumatol 2001;28:560-5.

19. Khan MA. Update on spondyloarthropathies. Ann Intern Med 2002;136:896-907.

20. Reveille JD, Ball EJ, Khan MA. HLA-B27 and genetic predisposing factors in spondyloarthropathies. Curr Opin Rheumatol 2001;13:265-72.

21. Gonzalez S, Garcia-Fernandez S, Martinez-Borra J, et al. High variability of HLA-B27 alleles in ankylosing spondylitis and related spondyloarthropathies in the population of northern Spain. Hum Immunol 2002;63:673-6.

22. Conde RA, Sampaio-Barros PD, Donadi EA, et al. Frequency of the HLA-B27 alleles in Brazilian patients with ankylosing spondylitis. J Rheumatol (submetido para publicação).

23. Smith JR, Rosenbaum JT. Management of uveitis. A rheumatologic perspective. Arthritis Rheum 2002;46:309-18.

24. Dougados M, Berenbaum F, Maetzel A, Amor B. Prévention des épisodes d'úvéite antérieure aigue des spondylarthropathies par la salazosulfapyridine. Rev Rhum 1993;60:81-3.

25. Benitez-del-Castillo JM, Garcia-Sanchez J, Iradier T, Bañares A. Sulfasalazine in the prevention of anterior uveitis associated with ankylosing spondylitis. Eye 2000;14:340-3.

26. Dougados M, Van der Linden S, Leirisalo-Repo M, et al. Sulfasalazine in the treatment of spondylarthropathies: a randomized, multicenter, double-blind, placebo-controlled study. Arthritis Rheum 1995;38:618-27.

27. Clegg DO, Reda DJ, Abdellatif M. Comparison of sulfasalazine and placebo for the treatment of axial and peripheral articular manifestations of the seronegative spondylarthropathies. Arthritis Rheum 1999;42:2325-9.

28. Sampaio-Barros PD, Costallat LTL, Bertolo MB, Marques Neto JF, Samara AM. Methotrexate in the treatment of ankylosing spondylitis. Scand J Rheumatol 2000;29:160-2.

29. Braun J, Brandt J, Listing J, et al. Treatment of active ankylosing spondylitis with infliximab: a randomized controlled multicentre trial. Lancet 2002;359:1187-93.

30. Van den Bosch F, Kruithof E, Baeten D, et al. Randomized doubleblind comparison of chimeric monoclonal antibody to tumor necrosis factor alfa (infliximab) versus placebo in active spondylarthropathy. Arthritis Rheum 2002;46:755-65.

31. Gorman JD, Sack KE, Davis Jr. JC. Treatment of ankylosing spondylitis by inhibition of tumor necrosis factor alfa. N Engl J Med 2002;346:1349-56

32. Smith JR, Levinson RD, Holland GN, et al. Differential efficacy of tumor necrosis factor inhibition in the management of inflammatory eye disease and associated rheumatic disease. Arthritis Care Res 2001;45:252-7.

33. Reiff A, Takei S, Sadeghi S, et al. Etanercept therapy in children with treatment-resistant uveitis. Arthritis Rheum 2001;44:1411-5. 